\title{
Wang-Landau Sampling of an Asymmetric Ising Model: A Study of the Critical Endpoint Behavior
}

\author{
Shan-Ho Tsai ${ }^{a, b}$, Fugao Wang ${ }^{a, *}$, and D.P. Landau ${ }^{a}$ \\ ${ }^{a}$ Center for Simulational Physics, University of Georgia, Athens, GA 30602, USA \\ ${ }^{b}$ Enterprise Information Technology Services, University of Georgia, Athens, GA 30602, USA
}

Received on 6 October, 2005

\begin{abstract}
We use the Wang-Landau algorithm to calculate a density of states for an asymmetric Ising model on a triangular lattice with two- and three-body interactions in an external field. An accurate density of states allows us to determine the phase diagram and to study the critical behavior of this model at and near the critical endpoint. We observe a divergence of the curvature of the spectator phase boundary at the critical endpoint in accordance with theoretical predictions.
\end{abstract}

Keywords: Critical endpoint; Wang-Landau sampling; Ising model; Triangular lattice; Three-body interaction

\section{INTRODUCTION}

A critical endpoint (CE) is a point in the phase diagram where a critical line meets and is truncated by a first-order transition line. Critical endpoints appear in the phase diagram of many physical systems such as binary fluid mixtures, superfluids, binary alloys, liquid crystals, certain ferromagnets, etc. The bulk critical exponents are believed[1] to be unchanged at $\mathrm{CE}$, but this has not been checked beyond phenomenological theory and renormalization calculations.[2] (However, the universal character of a double critical endpoint has been numerically determined from extensive Monte Carlo simulations [3].) Moreover, a new singularity in the first-order phase transition line was shown to arise at CE. [1,4] This prediction was confirmed by Fisher and Barbosa's phenomenological studies on an exactly solvable spherical model[4].

The critical endpoint behavior in a symmetrical binary fluid mixture was studied by Wilding[5] using a large scale Monte Carlo simulation[6] with multicanonical[7] and histogram reweighting[8] methods. He showed the first numerical evidence of a singularity at $\mathrm{CE}$ for the diameter of the liquid-gas coexistence curve and for the curvature of the spectator phase boundary.

In this paper we study a two-dimensional Ising model on a triangular lattice with two- and three-body interactions in a uniform external field. For a range of coupling parameters, this model has a critical endpoint.[9] We use the WangLandau sampling technique $[10,11]$ with a two-dimensional random walk to determine the density of states and thereby the phase diagram with great accuracy. The high resolution of this simulation allowed us to compute the curvature of the first-order phase transition line and observe the singularity that arises at CE. We were also able to determine the critical exponents along the second-order transition line and we confirm that these exponents indeed do not change as $\mathrm{CE}$ is approached.

*Current address: Intel Corporation, PC1-105, 44235 Nobel Drive, Fremont, CA 94538

\section{MODEL AND METHODS}

The model considered here is described by the Hamiltonian

$$
\mathcal{H}=-J \sum_{<i j>} S_{i} S_{j}-P \sum_{<i j k>} S_{i} S_{j} S_{k}-H \sum_{i} S_{i}
$$

where $S_{i}= \pm 1$ is an Ising spin on site $i$ of a two-dimensional triangular lattice, $\langle i j\rangle$ denotes pairs of nearest-neighbor spins, and $\langle i j k\rangle$ represents the three spins on the elementary triangles. The parameters $J$ and $P$ are two- and three-body nearest-neighbor spin couplings, respectively, and $H$ is an external magnetic field. The model is asymmetric because the Hamiltonian is not invariant when $H \rightarrow-H$. Periodic boundary conditions are used in this work.

Previous Monte Carlo simulations by Chin and Landau [9] showed that this model exhibits critical endpoints for a range of values of parameters $J$ and $P$. In particular, there is a critical endpoint in the $T$ and $H$ phase diagram when $J=1$ and $P=2$; this is the set of parameters used in this paper. We perform Wang-Landau sampling[10, 11] to determine the phase diagram of this model and to study its behavior at the phase transition lines and at the critical endpoint.

Unlike conventional Monte Carlo methods[6] that directly generate a canonical distribution at a given temperature $T$, the Wang-Landau method estimates the density of states directly and accurately via a random walk that produces a flat histogram in the random walk parameter space. Wang-Landau sampling has proven very useful and efficient in many different applications, including studies of complex systems with rough energy landscapes. For example, this method has been used in studies of a Potts antiferromagnet[12], random spin systems[13], quantum systems[14-16], fluids[17, 18], binary Lennard-Jones glass[19], liquid crystals[20], polymers[17, 21], proteins [22, 23], other molecular systems [24, 25], atomic clusters[26], optimization problems[27], and combinatorial number theory[28]. Generalizations and further studies of this sampling technique have also been carried out by several authors[29-33].

To overcome the barriers in both energy and magnetization spaces for the asymmetric Ising model, we have to perform a two-dimensional (2D) random walk as it was done in the spin 
glass problem [11]. We restrict $P / J$ to an integer $m(m=2$ in this paper); therefore we can rewrite the Hamiltonian in Eq. (1) as a sum of two parts,

$$
\begin{aligned}
\mathcal{H} & =-J E^{\prime}-H M^{\prime} \\
E^{\prime} & =\sum_{<i j>} S_{i} S_{j}+m \sum_{<i j k>} S_{i} S_{j} S_{k} \\
M^{\prime} & =\sum_{i} S_{i}
\end{aligned}
$$

where $E^{\prime}$ is proportional to the energy due to the two- and three-body interactions, and $M^{\prime}$ is the magnetization of the system. We perform a $2 \mathrm{D}$ random walk in the $E^{\prime}-M^{\prime}$ space using the Wang-Landau algorithm $[10,11]$ to estimate the density of states $g\left(E^{\prime}, M^{\prime}\right)$, which is defined as the number of spin configurations for any given $E^{\prime}$ and $M^{\prime}$. The estimate for $g\left(E^{\prime}, M^{\prime}\right)$ is improved at each step of the random walk, using a carefully controlled modification factor, to produce a result that converges to the real value very quickly, even for large system sizes. The thermodynamic quantities can then be extracted by applying canonical average formulas in statistical physics.

We provide a succinct description of the sampling method here, more details can be found elsewhere[10, 11]. At the start of the simulation, the density of states is unknown, so we simply set $g\left(E^{\prime}, M^{\prime}\right)=1$ for all possible $\left(E^{\prime}, M^{\prime}\right)$. Then we begin our random walk in $E^{\prime}-M^{\prime}$ space by choosing a site randomly and flipping its spin with a probability proportional to the inverse of the density of states. If we denote $A^{\prime}\left(E^{\prime}, M^{\prime}\right)$ and $A^{\prime \prime}\left(E^{\prime \prime}, M^{\prime \prime}\right)$ as the points before and after a spin is flipped, respectively, the transition probability from $A^{\prime}$ to $A^{\prime \prime}$ is:

$$
p\left(A^{\prime} \rightarrow A^{\prime \prime}\right)=\min \left[\frac{g\left(A^{\prime}\right)}{g\left(A^{\prime \prime}\right)}, 1\right] .
$$

If the point $A^{\prime \prime}\left(E^{\prime \prime}, M^{\prime \prime}\right)$ is accepted we multiply the existing density of states by a modification factor $f>1$, that is, $g\left(E^{\prime \prime}, M^{\prime \prime}\right) \rightarrow f g\left(E^{\prime \prime}, M^{\prime \prime}\right)$, and we update the histogram $h$ of visited states, that is $h\left(E^{\prime \prime}, M^{\prime \prime}\right) \rightarrow h\left(E^{\prime \prime}, M^{\prime \prime}\right)+$ 1. If $A^{\prime \prime}\left(E^{\prime \prime}, M^{\prime \prime}\right)$ is not accepted, we update $g\left(E^{\prime}, M^{\prime}\right) \rightarrow$ $f g\left(E^{\prime}, M^{\prime}\right)$ and $h\left(E^{\prime}, M^{\prime}\right) \rightarrow h\left(E^{\prime}, M^{\prime}\right)+1$.

We continue performing the random walk until the histogram $h\left(E^{\prime}, M^{\prime}\right)$ is flat in $E^{\prime}-M^{\prime}$ space. The modification factor $f$ introduces a systematic error for the estimated density of states. The magnitude of this error for $\ln \left[g\left(E^{\prime}, M^{\prime}\right)\right]$ is proportional to $\ln (f)$. To reduce this source of error, we systematically reduce the modification factor to a finer one using a function like $f_{i+1}=f_{i}^{1 / n}(n>1)$ where $i$ here denotes the number of iterations in the algorithm (each iteration is a $2 \mathrm{D}$ random walk in the $E^{\prime}-M^{\prime}$ space). To be explicit, when iteration $i$ generates a flat histogram, we reset the histogram to zero, and begin the next iteration with a new factor $f_{i+1}$. We end the simulation when the modification factor is smaller than a predefined value (such as $f_{\text {final }}=\exp \left(10^{-8}\right) \simeq 1.00000001$ used here). To speed up the convergence of the density of states to the true value, the initial modification factor was as large as $f=f_{0}=e \simeq 2.71828 \ldots$, and $n=4$ in our simulations. We perform the random walk on all possible $\left(E^{\prime}, M^{\prime}\right)$ space with a single computer processor.
It takes from about a few minutes to 2 days on a $1.3 \mathrm{GHz}$ Itanium processor to obtain $g\left(E^{\prime}, M^{\prime}\right)$ for the lattices with linear sizes $L=6$ to $L=30$ used here.

We should point out that it is impossible to obtain a perfectly flat histogram. A histogram may be considered flat if $h\left(E^{\prime}, M^{\prime}\right)$ for all possible $\left(E^{\prime}, M^{\prime}\right)$ is not less than $x \%$ of the average histogram, where $x \%$ is chosen according to the size and complexity of the system and the desired accuracy of the density of states. However, in this work we use a less stringent criterion: the histogram is considered flat when the number of entries larger than or equal to 2000 remains unchanged for $L^{2} \times 10^{6}$ spin flip trials.

With an accurate estimate of the density of states $g\left(E^{\prime}, M^{\prime}\right)$, we can calculate thermodynamic quantities at any temperature $T$, external magnetic field $H$, and coupling $J$ for the system with Hamiltonian given in Eq.(1). The spontaneous magnetization per lattice site as a function of $T$ and $H$ is given by

$$
M(T, H)=\langle M\rangle=\frac{\sum_{E^{\prime}, M^{\prime}} M^{\prime} g\left(E^{\prime}, M^{\prime}\right) e^{-\left(-J E^{\prime}-H M^{\prime}\right) / k_{\mathrm{B}} T}}{N \sum_{E^{\prime}, M^{\prime}} g\left(E^{\prime}, M^{\prime}\right) e^{-\left(-J E^{\prime}-H M^{\prime}\right) / k_{\mathrm{B}} T}}
$$

where we assume $J=1>0$ (ferromagnetic coupling), $N=L^{2}$ is the number of lattice sites; and $T$ is in unit of $1 / k_{\mathrm{B}}\left(k_{\mathrm{B}}\right.$ is the Boltzmann constant).

The susceptibility $\chi(T, H)$ also can be calculated from the density of states by:

$$
\chi(T, H)=d M / d H=N\left(\left\langle M^{2}\right\rangle-\langle M\rangle^{2}\right) / k_{\mathrm{B}} T .
$$

\section{RESULTS}

Figure 1 shows the phase diagram in the $T$ an $H$ plane for a finite lattice with $L=30$ (and the reader should remember that the entire phase diagram was obtained from a single simulation). There are three distinct phases with different values of magnetization at $T=0$ : ferromagnetic phase $\mathrm{A}(+++)$ with $M(0, H)=1$ (all spins are up), ferromagnetic phase $\mathrm{B}(--$ ) with $M(0, H)=-1$ (all spins are down) and ferrimagnetic phase $\mathrm{C}(--+)$ with $M(0, H)=-1 / 3$ (the spins on two sublattices are down and on the other are up).

The transitions between A and B as well as between A and $\mathrm{C}$ are first order, whereas the phase transition between $\mathrm{B}$ and $\mathrm{C}$ is second order. The critical endpoint, $(T, H)_{\mathrm{CE}}$, is defined as the point where the critical line $T_{c}(H)$ separating phases B and $\mathrm{C}$ meets, and is truncated by the first-order transition line $H_{\sigma}(T)$ separating phase A from phases B and C. The $H_{\sigma}(T)$ line is also called the spectator phase boundary. At the end of $H_{\sigma}(T)$ is a critical point $(T, H)_{\mathrm{c}}$. Our simulational results $T_{\mathrm{c}}(H=-6)=0$ and $H_{\sigma}(T=0)=-3$ are consistent with the exact solutions at zero temperature and early simulational data [9].

Though the spontaneous magnetization is a good parameter to characterize the three different phases, $M(T, H)$ is not the order parameter for the phase transitions because it does not vanish at any phase. For the transition between $\mathrm{A}$ and $\mathrm{C}$ phases as well as the transition between $B$ and $C$ phases, we 


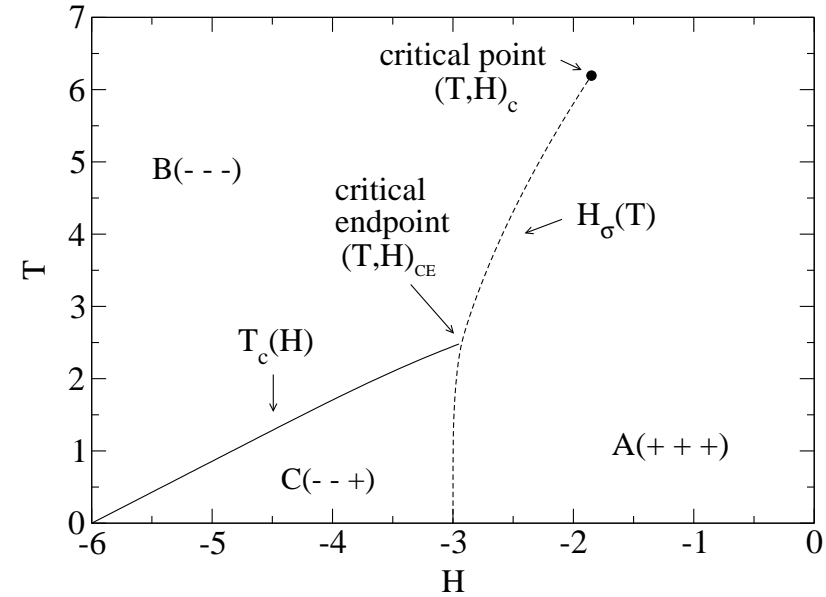

FIG. 1: Phase diagram for $L=30$.

define order parameters that are similar to those used for the $Q=3$ Potts model [34], because the degeneracy of the ground state in $\mathrm{C}$ phase is 3 . We define two components $\left(P_{1}, P_{2}\right)$ and a vector order-parameter $P$ from the magnetizations per site of the three sublattices $M_{1}, M_{2}$ and $M_{3}$ :

$$
\begin{aligned}
P_{1} & =\frac{1}{2}\left(M_{1}-\frac{M_{2}+M_{3}}{2}\right) \\
P_{2} & =\frac{\sqrt{3}}{4}\left(M_{2}-M_{3}\right) \\
P & =\sqrt{P_{1}^{2}+P_{2}^{2}}
\end{aligned}
$$

$P_{1}(T, H), P_{2}(T, H)$ and $P(T, H)$ approach zero in the phases $\mathrm{A}(+++)$ and $\mathrm{B}(---)$, and finite values in the ferrimagnetic phase $\mathrm{C}(--+)$.

To calculate thermodynamic quantities $P(T, H)$, we have to accumulate microscopic $P\left(E^{\prime}, M^{\prime}\right)$ during the random walk in the $E^{\prime}-M^{\prime}$ space. If $P\left(E^{\prime}, M^{\prime}\right)$ is the microscopic average value during a random walk at $\left(E^{\prime}, M^{\prime}\right)$, the thermodynamic quantity $P(T, H)$ can be calculated as:

$$
P(T, H)=\frac{\sum_{E^{\prime}, M^{\prime}} P\left(E^{\prime}, M^{\prime}\right) g\left(E^{\prime}, M^{\prime}\right) e^{-\left(-J E^{\prime}-H M^{\prime}\right) / k_{\mathrm{B}} T}}{\sum_{E^{\prime}, M^{\prime}} g\left(E^{\prime}, M^{\prime}\right) e^{-\left(-J E^{\prime}-H M^{\prime}\right) / k_{\mathrm{B}} T}} .
$$

The phase diagram in Fig. 1 is determined by the locations where the susceptibility $\chi_{p}(T, H)=d P / d H$ has the maximum value for a given value of $T$ for a lattice with $L=30$.

We determine the critical line $T_{c}(H)$ separating phase B and $\mathrm{C}$ with great accuracy, except when this line reaches CE. It is very difficult to determine $T_{c}(H)$ near CE, because the firstorder transitions are so strong. However, $T_{c}(H)$ is a smooth line and we extend it from low values of $H$ to where it meets the first-order transition line. We then take this meeting point as an estimate of the critical endpoint for the finite lattice $L$. For $L=30$ we estimate the critical endpoint as $(T, H)_{C E}=$ $(2.48 \pm 0.02,-2.93 \pm 0.02)$; this value will be used in the data analysis below.
The critical line $T_{c}(H)$ for this asymmetric Ising model studied here should be in the same universality class as the two-dimensional $Q=3$ Potts model, because the two models have the same symmetry. We recall that the conjectured values[35] for the critical exponents of the latter model are $\alpha=1 / 3, \beta=1 / 9, \gamma=13 / 9$, and $v=5 / 6$. In order to verify that our data are consistent with these conjectured exponents, we study finite size scaling of derivatives of the order parameter along $T_{c}(H)$. Because the order-parameter $P$ behaves as $P(T) \propto\left|\left(T-T_{\mathrm{c}}(H)\right) / T_{\mathrm{c}}(H)\right|^{\beta}$ near the critical line $T_{\mathrm{c}}(H)$, the finite-size behavior of $d P / d T$ at $T_{\mathrm{c}}(H)$ is

$$
\left.\frac{d P(L)}{d T}\right|_{T_{\mathrm{c}}(H)} \propto L^{(1-\beta) / \nu}
$$

The finite-size scaling behavior of the susceptibility $d P / d H$ at the critical line is

$$
\left.\frac{d P(L)}{d H}\right|_{T_{\mathrm{c}}(H)} \propto L^{\gamma / \nu}
$$

Figure 2(a) shows $d P / d T$ along $T_{c}(H)$ for several lattice sizes. Characteristic error bars shown for the $L=30$ data are obtained from several independent runs. In the inset of Fig.2(a), the absolute value of $d P / d T$ versus $L$ at three critical points in a wide region of external field $(H=-4.8,-4.0$ and -3.2) can be fitted well to the scaling form in Eq.(8) with $(1-\beta) / v=48 / 45 \simeq 1.07$, where we used the conjectured critical exponents for the $2 \mathrm{D} Q=3$ Potts model [35].

The susceptibility $d P / d H$ along $T_{c}(H)$ is shown in Fig.2(b) for several values of $L$. The finite-size behavior of $d P / d H$ in the inset of Fig.2(b) for different values of $H$ scale well according to the relation given in Eq.(9), using the $2 \mathrm{D} Q=3$ Potts critical exponents $\gamma / v=(13 / 9) /(5 / 6) \simeq 1.73$. The finite-size scaling shown in the insets of Figs.2(a) and 2(b) confirms that the transition between phase $\mathrm{C}(--+)$ and phase $\mathrm{B}(---)$ is in the same universality class as the $2 \mathrm{D} Q=3$ Potts model. Moreover, these results show that the critical exponents do not change when we approach the critical endpoint along the critical line $T_{\mathrm{c}}(H)$.

For the first-order transitions along $H_{\sigma}(T)$, finite-size analysis indicates that both at and away from CE the maximum of thermodynamics quantities are proportional to $L^{d}$ for finite-size systems (where $d$ is the dimensionality of the lattice). In particular, the susceptibility $\chi=d M / d H$ and the specific heat $C$ scale as

$$
\begin{aligned}
& \left.\chi(L)\right|_{H_{\sigma}(T)} \propto L^{d} \\
& \left.C(L)\right|_{H_{\sigma}(T)} \propto L^{d}
\end{aligned}
$$

Figures 3(a) and 3(b) show the susceptibility and the specific heat, respectively, along $H_{\sigma}(T)$ for several lattice sizes. The insets in these figures confirm that the scaling relations in Eq.(10) are satisfied both at $\mathrm{CE}$ and away from it (for example, at $\left.T=1.6 T_{C E}\right)$.

With an accurate estimate of the phase boundaries, we can calculate the curvature of the spectator phase boundary $H_{\sigma}(T)$. In Fig. 4, the curvature $d^{2} H_{\sigma}(T) / d T^{2}$ of the spectator 

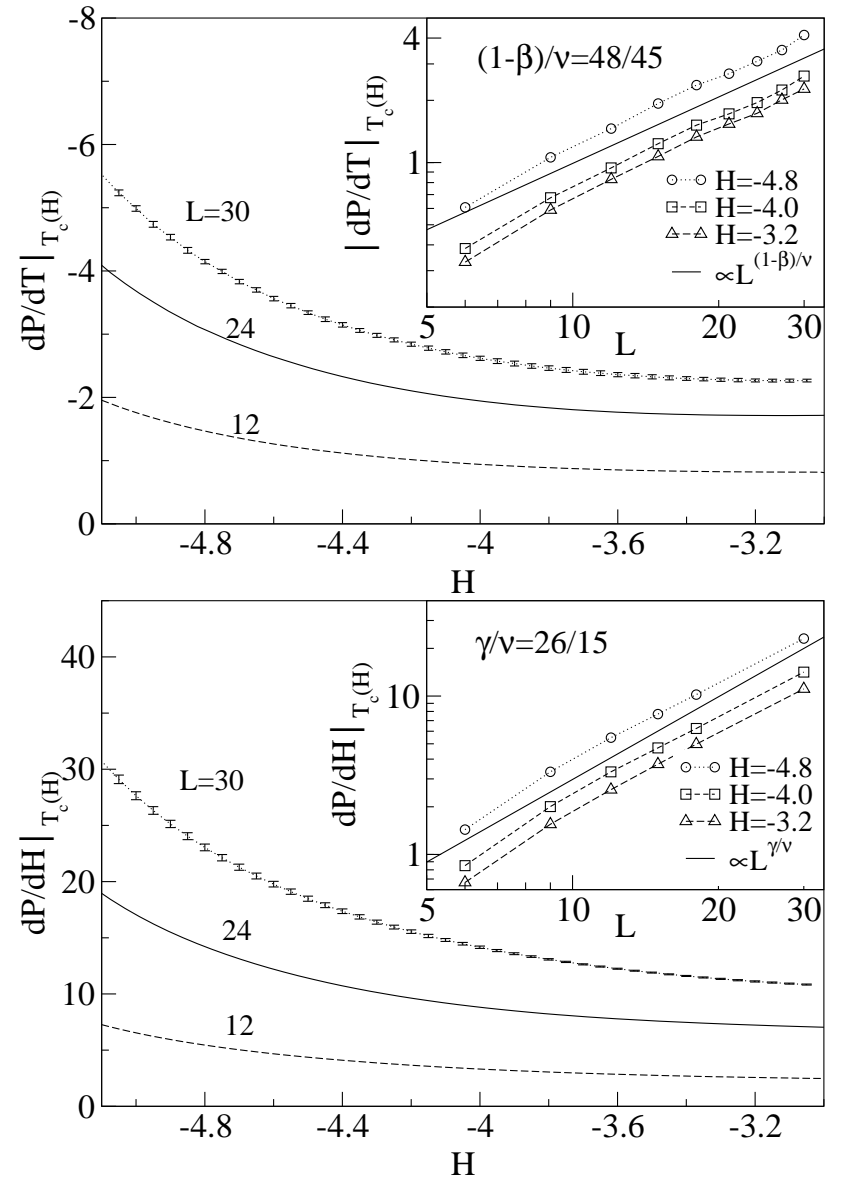

FIG. 2: Dependence of (a) $d P / d T$ and (b) $d P / d H$ on $H$ along the critical line $T_{c}(H)$ for several lattice sizes. The insets show the singularity in the respective thermodynamic quantities for three values of $H$ along $T_{c}(H)$. The error bars in the insets are not larger than the symbol sizes.

phase boundary has a very clear singularity at CE. According to a general finite-size scaling argument $[1,5]$, this curvature diverges at $\mathrm{CE}$ as

$$
\left.\frac{d^{2} H_{\sigma}(T, L)}{d T^{2}}\right|_{\mathrm{CE}}=c L^{\alpha / v}
$$

where $\alpha$ and $v$ are critical exponents defined on the critical line $T_{\mathrm{c}}(H)$. The lattice sizes used here are not large enough to give a good estimate of the scaling exponent $\alpha / v$ predicted by theory. For a binary fluid mixture, Wilding[5] observed a clear divergence of the curvature of the spectator phase boundary; however the system sizes he considered in his simulations were also not large enough to determine the finite-size scaling exponent accurately.

\section{CONCLUSION}

We used the Wang-Landau algorithm with a twodimensional random walk to determine the density of states
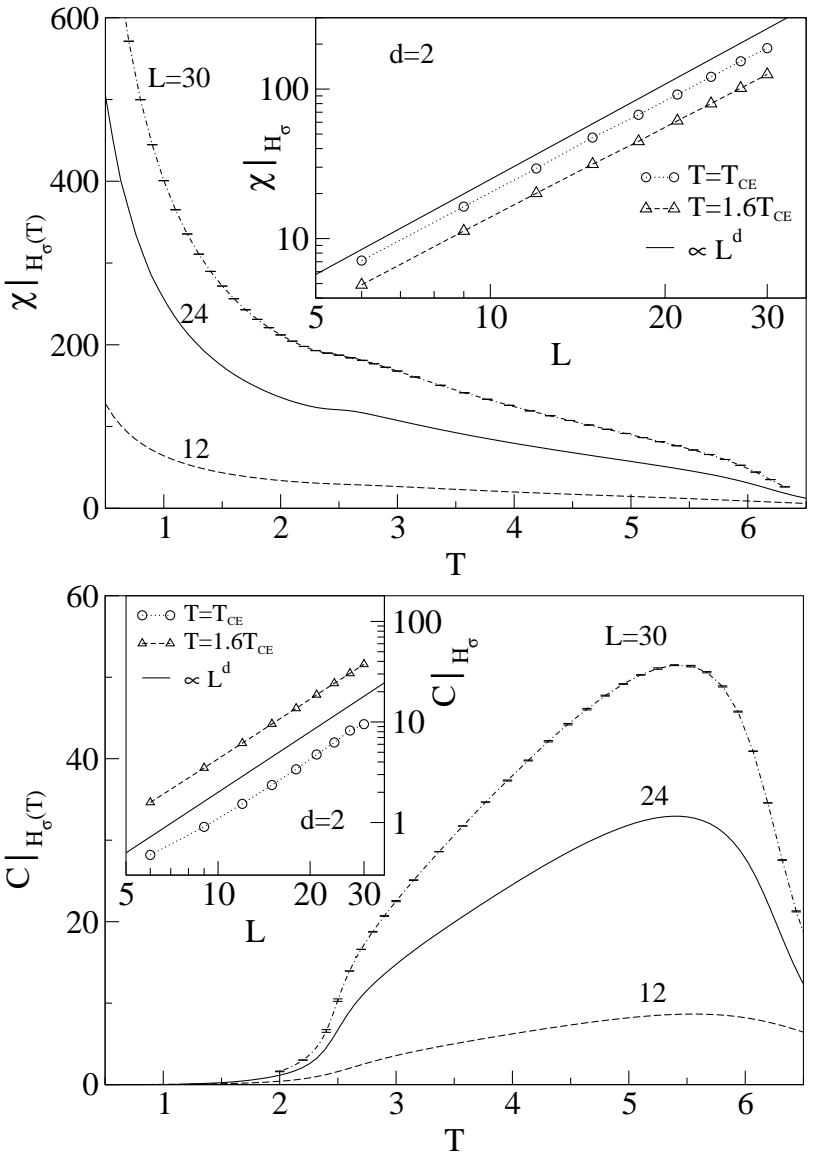

FIG. 3: Dependence of (a) the susceptibility $\chi=d M / d H$ and (b) the specific heat on $T$ along the first-order phase transition line $H_{\sigma}(T)$ for several lattice sizes. The insets show the singularity in the respective thermodynamic quantities at $T=T_{C E}$ and at $T=1.6 T_{C E}$ along $H_{\sigma}(T)$. The error bars in the insets are not larger than the symbol sizes.

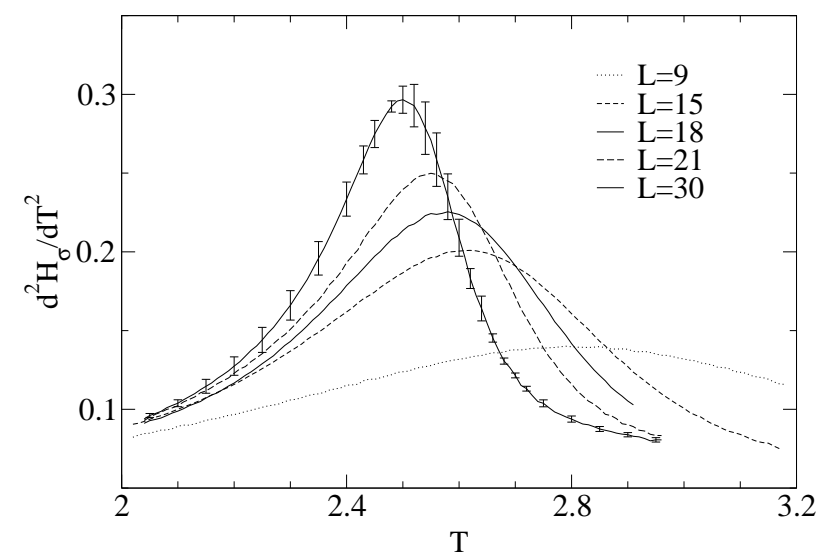

FIG. 4: Singularity of the curvature $d^{2} H_{\sigma} / d T^{2}$ of the spectator phase boundary $H_{\sigma}(T)$. 
$g\left(E^{\prime}, M^{\prime}\right)$ for an asymmetric Ising model with two- and threebody interactions on a triangular lattice, in the presence of an external field. With an accurate density of states we were able to map out the phase diagram accurately and observe a clear divergence of the curvature of the spectator phase boundary at the critical endpoint. However, larger systems have to be studied to obtain an accurate estimation of the scaling exponent of this divergence.

We show that the critical line separating phases $\mathrm{B}$ and $\mathrm{C}$ is indeed in the two-dimensional $Q=3$ Potts universality class and that the critical exponents do not change along the critical line. Thermodynamic quantities along the first-order line are shown to scale with lattice size as $L^{d}$ both at and away from the critical endpoint.

\section{Acknowledgments}

We thank J. Plascak, J. S. Wang, M. Müller, K. Binder, H. Meyer, and C. K. Hu for helpful discussions and comments. Part of the simulations were performed on the Itanium cluster at SDSC. This work is partially supported by the NSF under Grants number DMR-0341874 and DMR-0307082.
[1] M. E. Fisher and P. J. Upton, Phys. Rev. Lett. 65, 2402 (1990);65, 3405 (1990); M. E. Fisher, Physica A 172, 77 (1991).

[2] T. A. L. Ziman, D. J. Amit, G. Grinstein, and C. Jayaprakash, Phys. Rev. B 25, 319 (1982).

[3] J. A. Plascak and D. P. Landau, Phys. Rev E 67, 015103(R) (2003).

[4] M. E. Fisher and M. C. Barbosa, Phys. Rev. B 43, 11177 (1991); M. C. Barbosa and M. E. Fisher, Phys. Rev. B 43, 10635 (1991); M. C. Barbosa, Phys. Rev. B 45, 5199 (1992).

[5] N. B. Wilding, Phys. Rev. Lett. 78, 1488 (1997); Phys. Rev. E 55, 6624 (1997).

[6] D. P. Landau and K. Binder, A Guide to Monte Carlo Methods in Statistical Physics, second edition (Cambridge U. Press, Cambridge, 2005).

[7] B. A. Berg and T. Neuhaus, Phys. Rev. Lett. 68, 9 (1992).

[8] A. M. Ferrenberg and R. H. Swendsen, Phys. Rev. Lett. 61, 2635 (1988); 63, 1195 (1989).

[9] K. K. Chin and D. P. Landau, Phys. Rev. B 36, 275 (1987).

[10] F. Wang and D. P. Landau, Phys. Rev. Lett. 86, 2050 (2001); D. P. Landau and F. Wang, Comput. Phys. Commun. 147, 674 (2002); D. P. Landau, S.-H. Tsai, and M. Exler, Am. J. Phys. 72, 1294 (2004); D. P. Landau and F. Wang, Braz. J. Phys. 34 354 (2004).

[11] F. Wang and D. P. Landau, Phys. Rev. E. 64, 056101 (2001).

[12] C. Yamaguchi and Y. Okabe, J. Phys. A 34, 8781 (2001).

[13] Y. Okabe, Y. Tomita, and C. Yamaguchi, Comput. Phys. Commun. 146, 63 (2002).

[14] M. Troyer, S. Wessel, and F. Alet, Phys. Rev. Lett. 90, 120201 (2003).

[15] P. N. Vorontsov-Velyaminov and A. P. Lyubartsev, J. Phys. A 36, 685 (2003).
[16] W. Koller, A. Prüll, H. G. Evertz, and W. von der Linden, Phys. Rev. B 67, 104432 (2003)

[17] T. S. Jain and J. J. de Pablo, J. Chem. Phys. 118, 4226 (2003).

[18] Q. L. Yan, R. Faller, and J. J. de Pablo, J. Chem. Phys. 116, 8745 (2002).

[19] R. Faller and J. J. de Pablo, J. Chem. Phys. 119, 4405 (2003).

[20] E. B. Kim, R. Faller, Q. Yan, N. L. Abbott, and J. J. de Pablo, J. Chem. Phys. 117, 7781 (2002).

[21] T. S. Jain and J. J. de Pablo, J. Chem. Phys. 116, 7238 (2002).

[22] N. Rathore and J. J. de Pablo, J. Chem. Phys. 116, 7225 (2002).

[23] N. Rathore, T. A. Knotts, and J. J. de Pablo, J. Chem. Phys. 118, 4285 (2003).

[24] T. J. H. Vlugt, Mol. Phys. 100, 2763 (2002).

[25] F. Calvo, Mol. Phys. 100, 3421 (2002).

[26] F. Calvo and P. Parneix, J. Chem. Phys. 119, 256 (2003).

[27] M. A. de Menezes and A. R. Lima, Phys. A 323, 428 (2003).

[28] V. Mustonen and R. Rajesh, J. Phys. A 36, 6651 (2003).

[29] C. Yamaguchi and N. Kawashima, Phys. Rev. E 65, 056710 (2002).

[30] B. J. Schulz, K. Binder, and M. Müller, Int. J. Mod. Phys. C 13, 477 (2002).

[31] A. Huller and M. Pleimling, Int. J. Mod. Phys. C 13, 947 (2002).

[32] M. S. Shell, P. G. Debenedetti, and A. Z. Panagiotopoulos, Phys. Rev. E 66, 056703 (2002).

[33] B. J. Schulz, K. Binder, M. Müller, and D. P. Landau, Phys. Rev. E 67, 067102 (2003).

[34] Y. Tomita and Y. Okabe, Phys. Rev. Lett. 86, 572 (2001); K. Binder, K. Vollmayr, H.-P. Deutsch, J. D. Reger, M. Scheucher, and D. P. Landau, Int. J. of Mod. Phys. C 3, 1025 (1992).

[35] F. Y. Wu, Rev. Mod. Phys. 54, 235 (1982). 